\title{
BMJ Open School-based physical activity intervention for older adolescents: rationale and study protocol for the Burn 2 Learn cluster randomised controlled trial
}

\author{
Angus A Leahy, ${ }^{1,2}$ Narelle Eather, ${ }^{1,2}$ Jordan J Smith, ${ }^{1,2}$ Charles Hillman, ${ }^{3,4}$ \\ Philip J Morgan, ${ }^{1,2}$ Michael Nilsson, ${ }^{5}$ Chris Lonsdale, ${ }^{6}$ Ronald C Plotnikoff,, ${ }^{1,2}$ \\ Michael Noetel, ${ }^{6,7}$ Elizabeth Holliday, ${ }^{8}$ Tatsuya T Shigeta, ${ }^{3}$ Sarah A Costigan, ${ }^{1,2,9}$ \\ Frederick R Walker, ${ }^{10}$ Sarah Young, ${ }^{1,2}$ Sarah R Valkenborghs, ${ }^{1,10}$ Prajwal Gyawali, ${ }^{10}$ \\ Nigel Harris, ${ }^{11}$ Sarah G Kennedy, ${ }^{1,2}$ David R Lubans ${ }^{1,2}$
}

To cite: Leahy AA, Eather N, Smith JJ, et al. School-based physical activity intervention for older adolescents: rationale and study protocol for the Burn 2 Learn cluster randomised controlled trial. BMJ Open 2019;9:e026029. doi:10.1136/ bmjopen-2018-026029

- Prepublication history for this paper is available online To view these files, please visit the journal online (http://dx.doi org/10.1136/bmjopen-2018026029).

Received 17 August 2018 Revised 3 February 2019 Accepted 19 March 2019

Check for updates

(c) Author(s) (or their employer(s)) 2019. Re-use permitted under CC BY-NC. No commercial re-use. See rights and permissions. Published by BMJ.

For numbered affiliations see end of article.

Correspondence to

Professor David R Lubans;

david.lubans@newcastle.edu.au

\section{ABSTRACT}

Introduction This trial aims to investigate the impact of a school-based physical activity programme,

involving high-intensity interval training (HIIT), on the physical, mental and cognitive health of senior school students.

Methods and analysis The Burn 2 Learn (B2L) intervention will be evaluated using a two-arm parallel group cluster randomised controlled trial with allocation occurring at the school level (to treatment or wait-list control). Schools will be recruited in two cohorts from New South Wales, Australia. The trial will aim to recruit $\sim 720$ senior school students (aged 16-18 years) from 20 secondary schools (ie, 10 schools per cohort). A range of implementation strategies will be provided to teachers (eg, training, equipment and support) to facilitate the delivery of HIIT sessions during scheduled classes. In phase I and II (3 months each), teachers will facilitate the delivery of at least two HIIT sessions/week during lesson-time. In phase III (6 months), students will be encouraged to complete sessions outside of lesson-time (teachers may continue to facilitate the delivery of B2L sessions during lesson-time). Study outcomes will be assessed at baseline, 6 months (primary end point) and 12 months. Cardiorespiratory fitness (shuttle run test) is the primary outcome. Secondary outcomes include: vigorous physical activity, muscular fitness, cognition and mental health. A subsample of students will (i) provide hair samples to determine their accumulated exposure to stressful events and (ii) undergo multimodal MRI to examine brain structure and function. A process evaluation will be conducted (ie, recruitment, retention, attendance and programme satisfaction).

Ethics and dissemination This study has received approval from the University of Newcastle (H-20160424) and the NSW Department of Education (SERAP: 2017116) human research ethics committees.

Trial registration number ACTRN12618000293268; Preresults.

\section{Strengths and limitations of this study}

- Strengths of this study include the cluster randomised controlled trial design and adequate power to detect changes in primary and secondary outcomes.

- The measurement and analysis of the potential mechanisms responsible for the effects of physical activity on cognitive and mental health are strengths of this study.

- Focus on the factors influencing implementation and intervention fidelity are additional study strengths.

- As the majority of assessments will take place in the schools, it is not possible to assess fitness parameters using gold standard measures.

\section{INTRODUCTION}

Physical inactivity has been described as a global pandemic, ${ }^{1}$ and global estimates suggest that $<20 \%$ of adolescents are sufficiently active. ${ }^{2}$ Furthermore, physical activity declines dramatically during the teenage years ( 7\% each year from age 11 to 19 years $),{ }^{3}$ and Australian data indicate only $6 \%$ of older adolescents (15-17 years) are satisfying current physical activity guidelines of $60 \mathrm{~min}$ of moderate-to-vigorous physical activity per day. ${ }^{4}$ Recent data gathered from Australian secondary schools indicates that adolescent girls are less likely to meet physical activity recommendations in comparison to boys. ${ }^{5}$ Of additional concern, evidence suggests a decline in young people's cardiorespiratory fitness (CRF), which is an important predictor of overall health status, ${ }^{6}$ has occurred since the $1970 \mathrm{~s} .{ }^{78}$ While previous studies have noted small-to-moderate 
associations between physical activity and CRF in young people,$^{9}$ the relationship is stronger when activity of vigorous intensity is examined independently.

Adolescents who participate in physical activity of sufficient volume and intensity to improve their CRF will experience metabolic and cognitive benefits. For example, a recent longitudinal study involving a large sample of adolescents found that cardiometabolic risk declined in a dose-response manner with increasing vigorous physical activity in adolescents (healthy adolescents accumulated at least 7 min of vigorous activity each day), but not with increased volume of light or moderate physical activity. ${ }^{10}$ Both vigorous activity and CRF are also important for young people's mental ${ }^{11} 12$ and cognitive health. ${ }^{13} 14$ Recent systematic reviews have concluded that participating in physical activity can improve young people's cognitive control and academic performance, ${ }^{14}{ }^{15}$ but the underlying neurobiological, psychosocial and behavioural mechanisms not well understood. ${ }^{16}$

Schools are ideal settings for the delivery of physical activity programmes because they have access to young people as well as the necessary facilities and equipment, and availability of qualified staff. ${ }^{17}$ School-based physical activity interventions targeting children (aged 5-11 years) and young adolescents (aged 12-15 years) have had mixed success. ${ }^{18} 19$ Efficacy studies usually produce positive findings, but the promising findings from smallscale studies are rarely seen in large-scale effectiveness trials. ${ }^{20}$ Focusing on organisational change (ie, supportive school policies) and providing professional development for teachers can lead to improvements in physical activity and fitness in children ${ }^{21} 22$ and younger adolescents. ${ }^{23-26}$ However, it is relatively unknown if school-based interventions are effective with older adolescents (ie, senior school students [aged 16-18 years]) because few randomised controlled trials (RCTs) have been conducted with this population. ${ }^{19}$

Conducting and evaluating health promotion interventions with older adolescents is challenging, in part, due to the pressures associated with standardised testing at the end of secondary school and university entrance examinations. The focus on academic performance in the final years of secondary schooling and a lack of support from school administrators are major barriers to physical activity promotion in schools. ${ }^{172728}$ The success and sustainability of physical activity interventions is largely dependent on 'buy in' from school principals and teachers, which may wane over time in the face of competing time demands. As enhancing students' academic performance is the core business of schools, providing evidence for the impact of vigorous physical activity on cognitive and academic outcomes may provide a novel 'hook' for schools to implement physical activity interventions. Nevertheless, school-based physical activity interventions need to be time efficient because lack of time is the most commonly cited implementation barrier cited by teachers. ${ }^{27}$

High-intensity interval training (HIIT) is a time efficient strategy for improving metabolic health in adolescents and adults, ${ }^{29}{ }^{30}$ and typically consists of short, yet intense bouts of vigorous activity interspersed with brief periods of rest or light activity. Previous studies have shown HIIT can improve CRF (unstandardised mean difference $=2.6 \mathrm{~mL} / \mathrm{kg} / \mathrm{min}, 95 \% \mathrm{CI}=1.8$ to 3.3$)$, reduce body mass index (BMI, $-0.6 \mathrm{~kg} / \mathrm{m}^{2}, 95 \% \mathrm{CI}=-0.9$ to -0.4 ) and improve metabolic markers (ie, insulin sensitivity and fasting plasma insulin) in adolescents. ${ }^{30}{ }^{31}$ For most adolescents, the 'all out' maximal type of HIIT (ie, $100 \%$ of heart rate max) may not be palatable and such an approach has limited potential as a public health strategy. ${ }^{32}$ Alternatively, there is emerging evidence for the efficacy of less demanding HIIT protocols (eg, 85\% of heart rate max), although most experimental studies have been conducted in laboratory settings over short periods of time ( 8 weeks). ${ }^{31}$ Importantly, there is scope for developing novel HIIT protocols that retain the health-enhancing effects, and satisfy adolescents' desire for enjoyment and variety. ${ }^{33-35}$

We recently conducted the first 'teacher-facilitated' HIIT intervention for older adolescents, the Burn 2 Learn (B2L) pilot RCT. ${ }^{36}$ School teachers were asked to provide at least two opportunities during the school week for students $(n=68)$ to complete HIIT sessions during class time. The programme achieved high levels of recruitment $(85 \%)$ and retention $(90 \%)$ over the 14-week study period. Adherence to sessions was lower than prescribed (1.9 sessions/week during school) due to disruptions within the school (eg, examinations). Overall programme satisfaction was high among both students and teachers. Favourable intervention effects were found for CRF, lower-body muscular power (increases) and psychological distress (decrease) in the hypothesised directions. Our pilot study demonstrated that teachers can successfully facilitate the delivery of HIIT during the school day to improve older adolescents' fitness and wellbeing. However, it is unclear whether these positive findings can be replicated on a larger scale.

\section{Study objectives}

The primary aim of this trial is to determine the effect of the B2L intervention on older adolescents' CRF (primary outcome). Secondary outcomes of the trial include muscular fitness, body composition, mental health and cognitive control. This study will also test a range of potential neurobiological, psychosocial and behavioural mechanisms responsible for the effects of physical activity on cognitive and mental health. Finally, a detailed process evaluation will be conducted to determine if the intervention was delivered as intended and the factors influencing implementation.

\section{METHODS}

\section{Study design}

The trial is registered with the Australian New Zealand Clinical Trials Registry (ACTRN12617000544370) and the design, conduct and reporting will adhere 
to the Consolidated Standards of Reporting Trials ${ }^{37}$ and Template for Intervention Description and Replication $^{38}$ checklists. The B2L intervention will be evaluated using a two-arm parallel group cluster RCT with an intervention group and wait-list control group. Assessments will be conducted at baseline, 6 months (primary end point) and 12 months from baseline (secondary end point). The RCT will include two cohorts, one starting in 2018 (10 schools; 5 intervention and 5 control), and the other starting in 2019 (10 schools; 5 intervention and 5 control) and finishing in 2020. Baseline data collection will occur in the school term preceding the intervention delivery (ie, term 1 [February to April 2018 and 2019]). The intervention delivery will occur in terms 2 and 3 (May to September 2018, 2019). Post-test data collection (ie, 6-month follow-up) will commence midway through term 3 and continue until the end of term (August to September 2018 and 2019), with final follow-up assessments (ie, 12-month follow-up) being completed in term 1 of the following year (February to April 2019 and 2020).

\section{School recruitment and selection}

New South Wales (NSW) government secondary schools that include senior school students (ie, grades 11 and 12, students aged $16-18$ years) will be eligible to participate in the study. In cohort 1, eligible secondary schools located within 90 min drive from the University of Newcastle will be invited to participate. In cohort 2, eligible secondary schools located within 150 min drive from the University of Newcastle will be recruited. The selected geographical regions (ie, Hunter-Central Coast, Sydney, Northern Sydney, Western Sydney and New England) are broadly representative of urban and regional secondary schools in NSW.

Schools will be recruited via presentations at conferences and meetings (eg, regional meetings of the NSW Principals' Association) and emails sent directly to eligible schools (ie, school principals and grade 11 coordinators). Once schools have expressed an interest in the study, our Project Manager will meet with the school representative(s) and explain the study requirements. At this time, schools will be asked to identify a minimum of two grade 11 teachers willing to facilitate the delivery of scheduled B2L sessions during school hours. There are no restrictions regarding the teaching discipline (eg, mathematics, English, health and physical education) of grade 11 teachers eligible to participate in the study.

\section{Participants}

Two grade 11 teachers per school (B2L school champions) who agree to facilitate the delivery of B2L during scheduled class time. Eligible participants will be grade 11 students who are taught by one of the B2L school champions. Of note, students consenting to participate in the trial, are consenting to participate in the evaluation component (ie, completion of study measures). Students with a health or medical condition that would preclude participation in vigorous physical activity will be excluded from the study, but will still participate in normal lessons adapted by the B2L school champion. We will aim to recruit $\sim 36$ students (ie, 2 classes) per school.

\section{Sample size calculation}

Power calculations were based on the primary outcome of CRF, assessed using the Progressive Aerobic Cardiovascular Endurance Run (PACER) test. ${ }^{39}$ Baseline post-test correlation $(\mathrm{r}=0.90)$ and $\mathrm{SD}=29$ values were obtained from our pilot trial, and conservative intraclass correlation coefficient values of 0.20 and 0.03 were used to account for clustering at the class and school levels, respectively. ${ }^{40}$ To detect a clinically meaningful baseline-adjusted between-group difference of 6 laps ${ }^{3341}$ with $80 \%$ power at a $5 \%$ significance level will require 280 students per treatment group (ie, 2 classes of 14 students from each of 10 schools). Inflating the sample size to 18 students per class, or 360 students per treatment arm (ie, total sample of 720 students) allows for a potential drop-out rate of $20 \%$ at our primary study end point (ie, 6 months).

\section{Blinding and randomisation}

Randomisation will occur once 10 schools (cohort 1) have been recruited and completed baseline assessments. The same process will be repeated for cohort 2. Pairs of schools will be matched based on the following characteristics: geographic location (ie, region, rural/urban, coastal/inland), school area-level socioeconomic status (ie, using the Socio-Economic Indexes For Areas Index of Relative Socio-Economic Disadvantage), ${ }^{42}$ schools' student population educational advantage (ie, using the Index of Community Socio-Educational Advantage) and where possible, proposed class delivery (eg, mathematics, English and personal development, health and physical education). Schools will be randomised by an independent researcher using a computer-based random number generator, such that one school from each pair will be allocated to the intervention condition and the other to the control condition. Using this approach, each school will have an equal chance of being allocated to the intervention condition, while maintaining an appropriate balance of school characteristics across the two conditions. Schools randomised to the intervention condition will deliver the B2L programme during the study period, whereas schools allocated to the control condition will continue with usual school practice (ie, normal curricular lessons) for the duration of the study period (ie, until completion of 12-month study assessments). Schools allocated to control group will then receive the intervention following final study assessments (ie, the following year). The decision to use a wait-list control design, rather than an attention-matched placebo, was based on the following. First, the research team will have little contact with students, as the programme will be delivered by teachers during their regularly scheduled lessons. Second, for our findings to have greater external validity, it is important that our control group reflects 'usual practice'. Finally, based on our previous studies, a 
wait-list control group is acceptable for schools and the majority of our school-based trials have achieved high levels of retention $(80 \%-90 \%){ }^{244344}$

\section{Intervention delivery, components and implementation strategies}

The B2L intervention will be delivered in three phases: phase I) getting started, phase II) maintaining student interest and phase III) moving towards independence. In phases I and II (term 2-term 3; May-September 2018 and 2019), school champions will be tasked with facilitating the delivery of at least two HIIT sessions/week during lesson-time. During phase I, school champions will attend a 1-day professional learning workshop led by the research team. The workshop will focus on providing the school champions with the knowledge and skills needed to introduce students to HIIT and develop their competency. Phase II will involve a greater emphasis on student responsibility and control, and introduce additional intervention resources (ie, new HIIT task cards) to maintain student interest. In phase III (term 4/term 1; October-April 2018/2019 and 2019/2020), students will be encouraged to complete sessions outside of lesson-time (teachers may continue to facilitate the delivery of B2L sessions during lesson-time).

The HIIT sessions will involve a combination of aerobic (eg, shuttle runs, jumping jacks, boxing, dancing) and body weight muscle-strengthening exercises (eg, push-ups, squat jumps and walking lunges), and have been designed to be fun and engaging as well as vigorous in nature. Participants will be able to select from a variety of predesigned HIIT task cards which will be released across the phases of the programme to promote variety and sustain participant interest. Phase I) getting started: (i) Gym HIIT-combination of aerobic (eg, skipping) and strength exercises (eg, squat jumps), (ii) Sport HIITusing sports equipment (eg, shuttle run while dribbling a basketball), (iii) Class HIIT-exercises that can be performed in a standard classroom (eg, running on the spot, tricep dips), (iv) Quick HIIT—using Tabata protocol (eg, $20 \mathrm{~s}$ intense work, followed by $10 \mathrm{~s}$ rest). Phase II) maintaining student interest: (i) Hip-hop HIIT-high-intensity hip-hop dance movements, (ii) Combat HIITinvolves boxing/mixed martial arts movements (eg, front kicks), (iii) Brain HIIT—activities that encourage thinking while participating in high-intensity activity, (iv) Rumble HIIT-high-intensity rough and tumble exercises (eg, partner knee taps) combined with aerobic activity, (v) Custom HIIT-participants design their own HIIT workout. Phase III) moving towards independence. (i) Beach HIIT - using the natural coastal environment (eg, sand shuttle runs and surfer style get-ups), (ii) Park HIIT-exercise sessions adapted for the park setting (eg, triceps dips using park benches).

A common criticism of public health research is the development of interventions that are not 'scalable' and unlikely to be adopted and implemented in real-world settings. ${ }^{45}$ The B2L intervention has been designed in consultation with the NSW Department of Education and guided by the
Consolidated Framework for Implementation Research ${ }^{46}$ to maximise scalability and sustainability. A summary of the B2L intervention components are provided in table 1. The multicomponent intervention will target schools, principals, teachers, students and parents using: (i) an information seminar delivered by school champions, (ii) school-based physical activity sessions for two school terms, (iii) smartphone application and heart rate monitors and (iv) information for parents (ie, print or e-newsletters). A range of implementation strategies were designed to support the delivery of the B2L programme (table 2) and include the following: (i) intervention characteristics, (ii) outer setting (ie, educational authorities), (iii) inner setting (ie, schools), (iv) characteristics of teachers and (v) implementation process.

\section{Theoretical framework for the intervention}

Several theoretical frameworks have been used to guide intervention design. First, Beets et al theory of expanded, extended and enhanced opportunities ${ }^{47}$ suggests that the extension and enhancement of existing physical activity opportunities, as well as the creation of new opportunities (ie, expansion) are needed to increase youth physical activity levels. For the current study, the provision of an entirely new opportunity for physical activity (ie, expansion) was considered necessary, as the majority of secondary schools in NSW do not provide mandatory physical activity for senior students. During phases I and II of the intervention, the HIIT sessions will be facilitated during scheduled class-time, therefore adopting a compulsory application of theory of expanded, extended and enhanced opportunities. In the third phase, students will be encouraged to complete two to three sessions per week within or beyond the school day. During this phase, students will be encouraged to self-monitor their participation in HIIT sessions using the B2L smartphone app.

With reference to self-determination theory, B2L HIIT sessions have been developed with a focus on enhancing students' autonomous motivation for vigorous physical activity within and beyond the school setting by satisfying their basic psychological needs for autonomy (feeling in control), competence (feeling capable) and relatedness (feeling connected with others). ${ }^{48}$ Teachers will learn to facilitate the B2L sessions using the Supportive, Active, Autonomous, Fair and Enjoyable (SAAFE) physical activity delivery principles (table 3). ${ }^{49}$ Participants' need for autonomy will be satisfied by providing opportunities for choice within sessions (eg, type of activity, music playing and training partner) and explaining the rationale for the programme in an information seminar. The introductory seminar will reinforce the importance of exercise for cognitive health and academic performance, which may be salient outcomes for students during this period of their schooling. Competence will be satisfied using positive and specific feedback from teachers, an explicit focus on effort over performance (via heart rate feedback), and through the provision of resources designed to support the development of exercise skills. Teachers will be encouraged to 


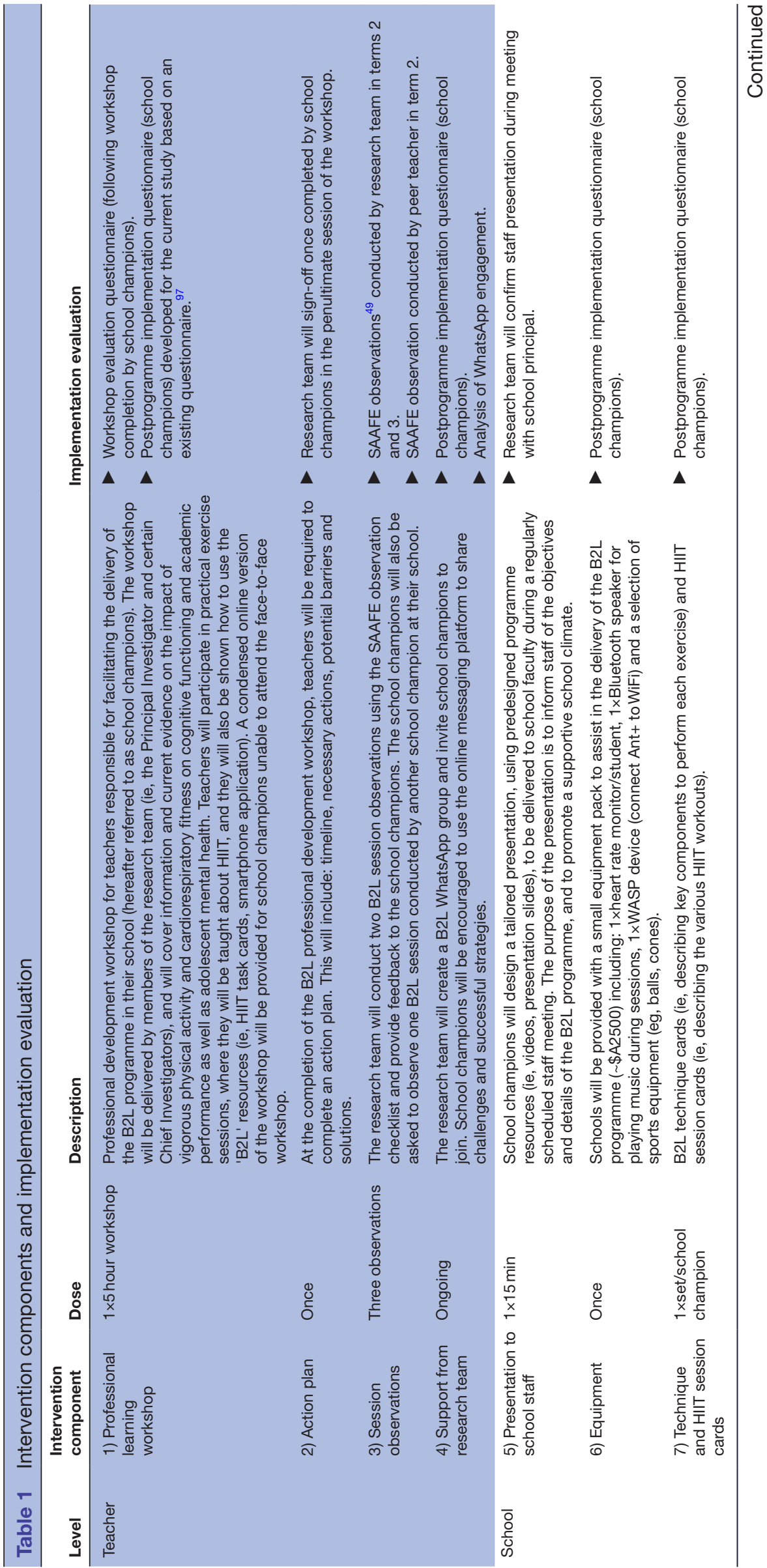




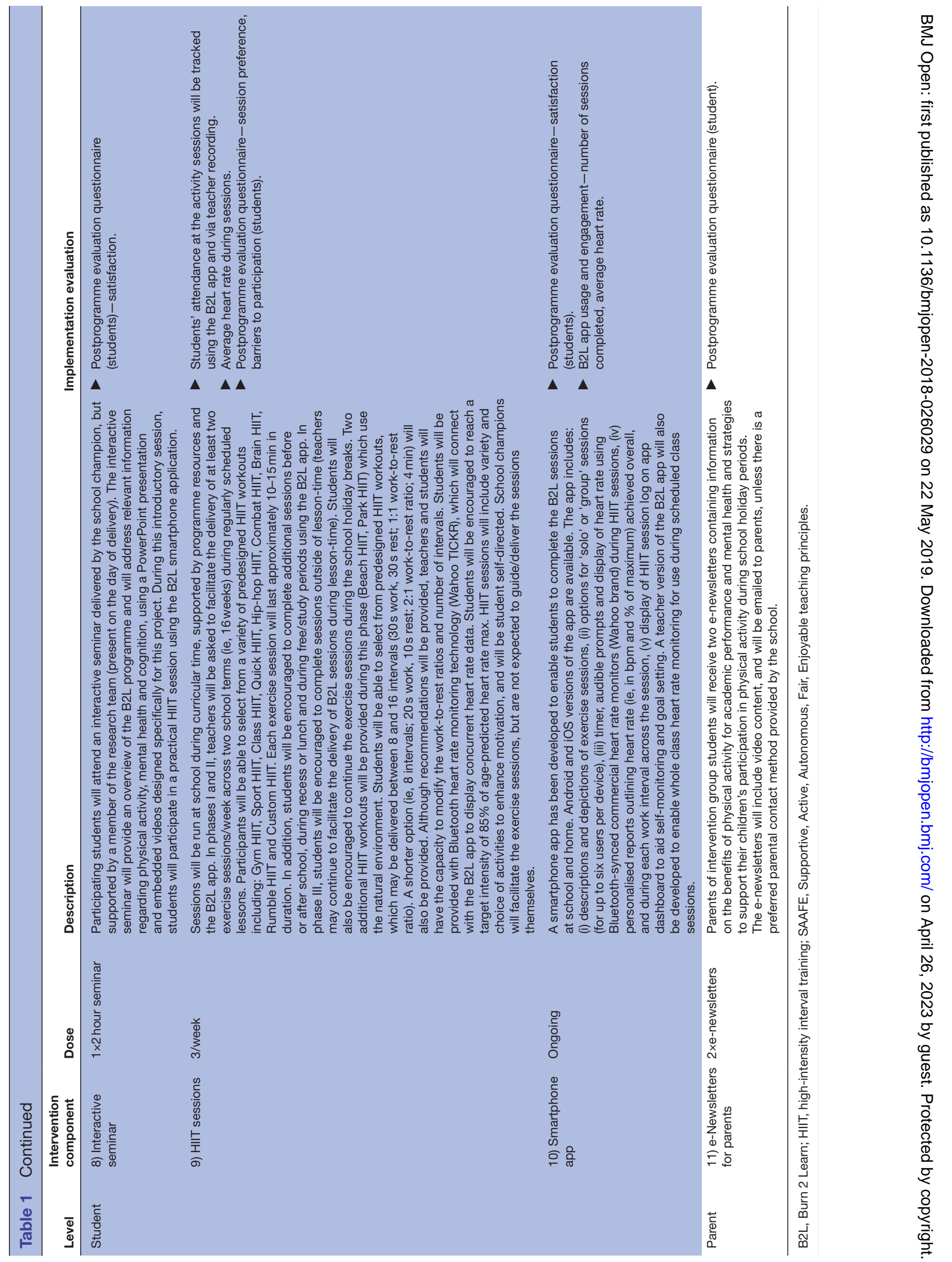


Table 2 Strategies used to facilitate implementation in the B2L intervention

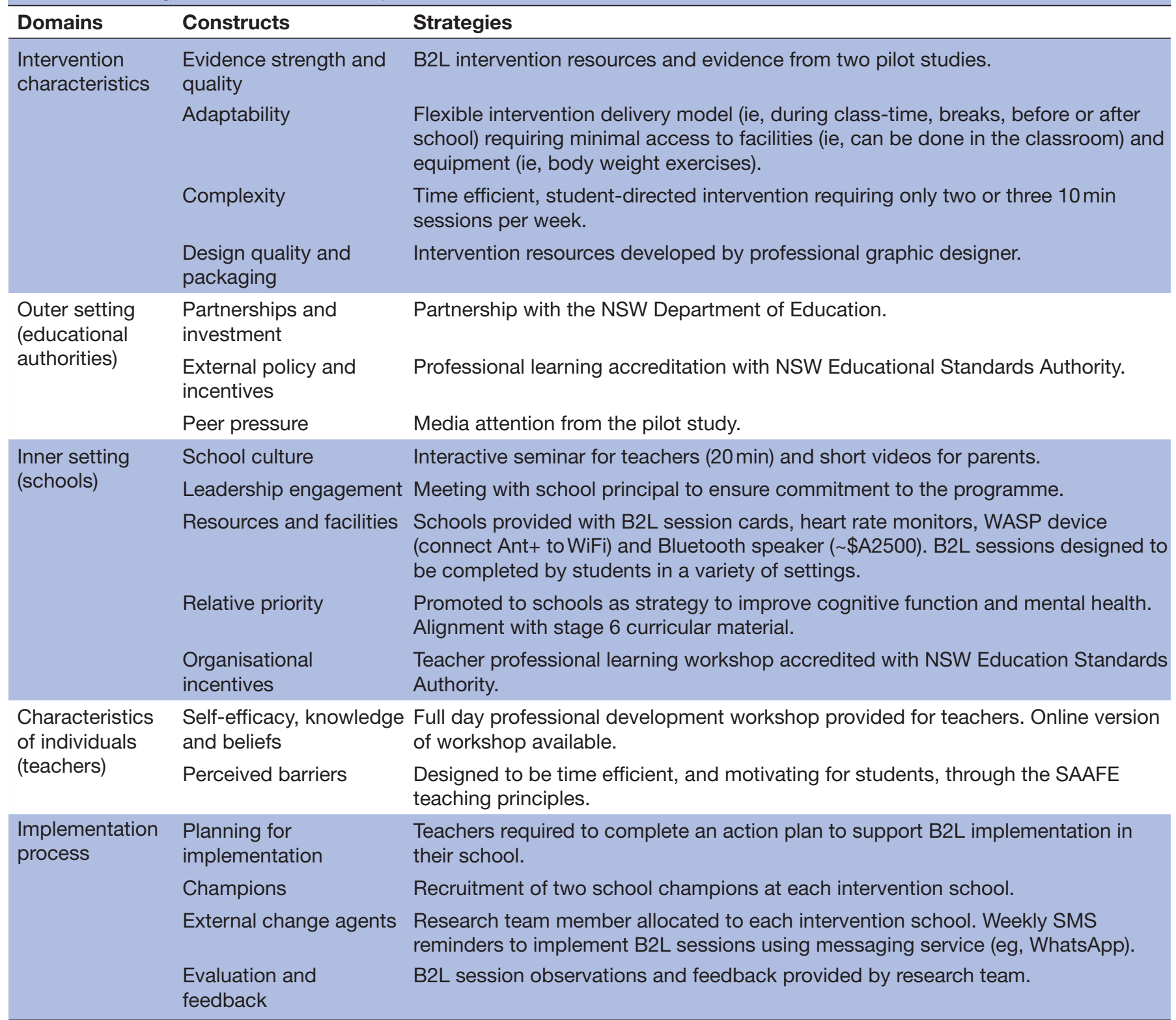

B2L, Burn 2 Learn; NSW, New South Wales; SAAFE, Supportive, Active, Autonomous, Fair, Enjoyable teaching principles.

adopt practices that support relatedness and group cohesion during HIIT sessions (ie, encouraging supportive behaviour among students such as 'high fives' and partner work). ${ }^{50}$

\section{Measures and data collection}

Apart from multimodal MRI, all assessments will be conducted at the study schools on the same day by trained research assistants, who will be blinded to group allocation at all time-points. Demographic information and self-report measures will be collected using electronic tablets under exam-like conditions. Cognitive testing will occur on university laptops and participants will receive instructions and practice prior to performing each of the cognitive tasks. Self-report and cognitive measures will occur prior to fitness assessments in a randomised order.
Anthropometric assessments will be conducted in a sensitive manner by same-sex research staff when possible. Research assistants will provide a brief verbal description and demonstration of each fitness test prior to commencement. The timing for participants to complete all measures is approximately $90 \mathrm{~min}$.

\section{Primary outcome}

Cardiorespiratory fitness

CRF will be assessed using the PACER FITNESSGRAM testing procedures. This test is the most widely accepted field-based measure of CRF, demonstrating high reliability and validity. ${ }^{51}$ A $20 \mathrm{~m}$ course will be set up on a hard surface with participants instructed to run back and forth between two sets of lines while keeping pace with a prerecorded cadence (indicated by a single beep for each 


\begin{tabular}{|c|c|c|}
\hline Principle & Definition & Example strategies \\
\hline Supportive & $\begin{array}{l}\text { Sessions are designed to facilitate a } \\
\text { supportive environment }\end{array}$ & $\begin{array}{l}\text { Provide constructive feedback. } \\
\text { Praise effort and improvement. } \\
\text { Encourage supportive behaviour among students. }\end{array}$ \\
\hline Active & Sessions are highly active & $\begin{array}{l}\text { Commence sessions quickly. } \\
\text { Minimise talk and instruction time. } \\
\text { Encourage students to exercise at high-intensity. }\end{array}$ \\
\hline Autonomous & Sessions involve elements of choice & $\begin{array}{l}\text { Provide students with opportunities of choice (eg, music, } \\
\text { partner, activity). } \\
\text { Minimise controlling language (eg, ordering students around). } \\
\text { Remind students about the benefits of high-intensity activity. }\end{array}$ \\
\hline Fair & $\begin{array}{l}\text { Sessions provide all students with } \\
\text { opportunities to experience success }\end{array}$ & $\begin{array}{l}\text { Encourage self-comparison rather than peer-comparison. } \\
\text { Encourage students to modify exercises to personal fitness } \\
\text { and ability level. } \\
\text { Treat all students equally and fairly (ie, high expectations for } \\
\text { all). }\end{array}$ \\
\hline Enjoyable & $\begin{array}{l}\text { Sessions are designed to be enjoyable and } \\
\text { engaging for all students }\end{array}$ & $\begin{array}{l}\text { Play motivational music during exercise sessions. } \\
\text { Provide students with a variety of HIIT workout options. } \\
\text { Encourage students to reflect on their postexercise affect (ie, } \\
\text { how they are feeling). }\end{array}$ \\
\hline
\end{tabular}

SAAFE, Supportive, Active, Autonomous, Fair, Enjoyable teaching principles.

$20 \mathrm{~m}$ shuttle). The test begins at a slow pace $(8.5 \mathrm{~km} /$ hour), and increases by $0.5 \mathrm{~km} /$ hour with each passing minute (as indicated by a triple beep). The test ends when participants fail to complete a shuttle (20 m lap) before the beep sounds, on two consecutive shuttles, or on volitional failure. Verbal encouragement will be provided by test administrators in order to maximise participant motivation. The last successful stage will be recorded and converted into the number of $20 \mathrm{~m}$ laps completed, which will constitute the primary outcome measure. The total number of laps will be used to estimate maximal aerobic capacity (ie, $\mathrm{VO}_{2}$ max), using the following equation: $45.619+(0.353 \times$ PACER laps $)-(1.121 \times$ age $) .{ }^{52}$ Estimated $\mathrm{VO}_{2}$ max will be used to classify participants into fitness zones according to criterion-referenced age-specific and sex-specific cut-offs developed by the Cooper Institute. ${ }^{53}$

\section{Secondary outcomes}

Physical activity

Participants will be instructed to wear an ActiGraph GT9X Link accelerometer on their non-dominant wrist for 24hours/day (even when bathing, swimming and sleeping) for a period of seven consecutive days. Weekday and weekend day (ie, mean minutes per day) physical activity will be calculated separately, using existing thresholds for categorising physical activity into light, moderate and vigorous intensity. ${ }^{54}$

\section{Muscular fitness}

Upper body muscular endurance will be assessed using the 90-degree push-up test. ${ }^{39}$ Using a cadence of 40 beats per minute, participants lower themselves in a controlled manner until a 90-degree angle is formed at the elbow before pushing back up. The test concludes when the participant either fails to lower themselves to the required depth on two non-consecutive repetitions (warning verbalised by assessor, repetitions counted), fails to maintain movement in time with the metronome, fails to maintain appropriate technique (back straight) or on volitional failure of the test. Lower body muscular power will be assessed using the standing long jump test. ${ }^{55}$ From a standing position behind a line marked at zero centimetres, participants perform a maximal long jump taking off and landing with two feet, simultaneously. The test will be performed twice, with the maximal distance jumped recorded as the participant's final score. Both measures of muscular fitness demonstrate high validity and reliability in adolescents. ${ }^{567}$

\section{Body composition}

Body weight and height will be measured using a portable digital scale (A\&D Medical UC-352-BLE Digital Scales) and a portable stadiometer (Seca 213 Portable Height Measuring Rod Stadiometer), respectively. Both weight and height will be measured twice to reduce the risk of measurement error. A third measurement will occur should there be a difference of $>0.1 \mathrm{~kg}$ for weight, and $>0.3 \mathrm{~cm}$ for height between the first and second measurement. BMI will be calculated using the standard formula (weight $[\mathrm{kg}] /$ height $\left[\mathrm{m}^{2}\right]$ ). Age-specific and sex-specific BMI z-scores will be calculated and participants will be classified into weight categories according to International Obesity Task Force cut-offs. ${ }^{58}$

\section{Cognitive control}

Using a laptop, specialised software (PsychoPy) ${ }^{59}$ will be used to assess working memory and inhibition. A modified version of an Eriksen flanker task will be used 
to measure inhibition, which is a reliable and valid measure in youth.$^{60}$ In this task, participants are required to respond (using specific keys) to the direction of the centrally presented arrow among either 'congruent' or 'incongruent' flanking arrows. Congruent trials consist of five horizontal arrows facing the same direction (ie, $<<<<<$ or $>\gg \gg>>$ ), while incongruent trials consist of the central arrow facing the opposite direction to the four flanking arrows $($ ie,$<<><<$ or $>><>>)$. Participants are challenged with a random sequence of congruent and incongruent trials and instructed to identify the direction of the centrally presented, target arrow as quickly and accurately as possible. Following instructions, participants will be presented with a practice block consisting of 25 trials to familiarise themselves with the test. If participants achieve below satisfactory overall accuracy $(<70 \%)$, they will complete another practice block of 25 trials, and the test administrator will confirm their understanding of the test. Participants then complete an experimental block consisting of 150 trials (with an equal distribution of congruent and incongruent). Stimuli are presented for $100 \mathrm{~ms}$, with a randomly allocated interstimulus interval of 900,1050 or $1200 \mathrm{~ms}$ separating each trial. Response time (ie, the time in ms taken by the participant to press the key associated with the direction of the target arrow) and response accuracy (ie, the percentage of trials for which the participant correctly indicated the direction of the target arrow) and will be recorded. An interference score (ie, the difference in performance outcomes between congruent and incongruent trials) will also be calculated for both accuracy and response time. A lower interference score demonstrates higher inhibitory control.

Working memory will be assessed using a serial n-back task, which is a widely used and reliable measure ${ }^{6162}$ Two task conditions (ie, 1-back and 2-back) will be evaluated which differ in the degree of cognitive demand. In these tasks, participants are presented with a series of six basic shapes (ie, square, star, circle, cross, crescent, triangle) and required to recall (using specific keys) whether the shape currently displayed (trial ' $n$ ') matches the shape immediately prior (1-back, 'n-1'), or two shapes prior (2-back, 'n-2'). For each trial (within each n-back condition), participants are required to indicate whether the shape is a match (ie, target) or not a match (ie, non-target). Shapes are presented for a duration of 250 ms following a fixed interstimulus interval of $2500 \mathrm{~ms}$. Following task instructions, participants will complete a practice block consisting of 20 trials to ensure understanding. If overall accuracy is below $70 \%$, participants will complete another practice block, and the test administrator will confirm their understanding of the test. Each task condition (ie, 1-back, 2-back) consists of two blocks of randomised target $(n=24)$ and non-target $(n=48)$ trials, and are presented in a counterbalanced order. Response time and accuracy will be recorded for both target shapes (ie, correctly identifying a match) and non-target shapes (ie, correctly identifying a non-match). Additionally, the relative proportion of 'hits' (correct target trials) to 'false alarms' (incorrect selection on target trial), otherwise known as the d-prime score, will be calculated for both task conditions. ${ }^{63}$ A higher d-prime score reflects a greater capacity to differentiate target from non-target shapes when performing the n-back tasks, thereby indicating greater working memory.

\section{Stress}

Objectively measured stress will be determined by examining the build-up of cortisol within the hair shaft using a sample taken from the vertex at the rear of the head. As human hair grows at a rate of approximately $1 \mathrm{~cm}$ per month, each $\mathrm{cm}$ is considered to reflect life stressors experienced by the individual over the last month. ${ }^{64}$ Cortisol concentrations will be determined from the $3 \mathrm{~cm}$ hair segment (maximum) most proximal to the scalp. This will represent the cumulative stress level in the past 2-3months. Interindividual variation in hair growth rate can be factored into analyses by measuring hair growth at the initial sampling site 1 month later. The intraindividual stability $(\mathrm{r}=0.68-0.79)^{65}$ and validity of hair cortisol as a retrospective index of stress are supported in the literature in adults ${ }^{667}$ and adolescents. ${ }^{68}$ Perceived stress will be self-reported using the Perceived Stress Scale, which is designed to assess the degree to which situations in one's life are stressful. ${ }^{69}$ Participants will be required to respond to the 10 -item questionnaire in relation to the previous month (eg, "In the last month, how often have you felt you were on top of things?"). Responses are scored on a 5-point scale ranging from 0 'never' to 4 'very often' and then summing across all scale items. Higher scores indicate a greater degree of subjective stress experienced by participants.

\section{Psychological difficulties}

The Strengths and Difficulties Questionnaire will be used to assess psychological distress. ${ }^{70}$ The questionnaire consists of 25 items, covering 2 subscales (ie, strengths and difficulties). The strengths subscale consists of one domain (prosocial behaviour (eg, "I try to be nice to other people. I care about their feelings"), and the difficulties subscale consists of four domains: emotional symptoms (eg, "I worry a lot"), conduct problems (eg, "I get very angry and often lose my temper"), hyperactivity (eg, "I am restless, I cannot stay still for long") and peer problems (eg, "I would rather be alone than with people of my own age"). For each item, participants respond using a 3-point scale (ie, 'not true' $=0$, 'somewhat true' $=1$ and 'certainly true' $=2$ ). For each of the five domains the score can range from 0 to 10 . A difficulties composite score will be obtained by adding the scores of all four difficulty domains, with a possible range from 0 to 40 . Lower scores indicate fewer psychological difficulties.

\section{Autonomous motivation}

Motivation for physical activity will be assessed using the 'Behavioural Regulations in Exercise Questionnaire'.71 The identified (eg, "I value the benefits of exercise"), and 
intrinsic (eg, "I exercise because it's fun") regulation subscales will be used. Responses are scored on a 5-point scale ranging from 0 'not true for me' to 4 'very true for me'.

\section{Basic psychological needs satisfaction}

Friends' and teachers' support for exercise will be assessed via the 'Adolescent Psychological Need Support in Exercise Questionnaire'. ${ }^{72}$ Items assess needs satisfaction during exercise across the three psychological needs identified within self-determination theory, namely autonomy support (eg, "I feel that they understand why I choose to exercise"), relatedness support (eg, "I feel they care about me") and competence support (eg, "They display confidence in my exercise ability"). Participants respond using a 7-point Likert scale ranging from 1 'strongly disagree' to 7 'strongly agree'.

\section{Psychological well-being}

Well-being is assessed using the validated Warwick-Edinburgh Mental Well-being Scale. ${ }^{73}$ The 14 -item questionnaire requires participants to reflect on their experiences over the last 2 weeks. Items are scored on a 5-point scale ranging from 1 'none of the time' to 5 'all of the time', and summed across all to produce a well-being composite (possible range $=14-70)$.

\section{Perceived fitness}

Perceived physical fitness will be self-reported using the International Fitness Scale (IFIS). ${ }^{74}$ Participants are required to report perceptions of their 'general fitness' and four specific subcomponents of health-related fitness. The 5-item instrument is scored on a scale ranging from 1 'very poor' to 5 'very good'. The validity and test-retest reliability of the IFIS has been found to be acceptable among a sample of youth aged 9-12 years. ${ }^{74}$

\section{HIIT self-efficacy}

Self-efficacy for HIIT will be assessed using a 6-item scale developed for the current study. The scale uses the common stem "If you really wanted to, how confident are you that you can..." and participants respond as follows: 1 'not at all confident' to 10 'completely confident'. Sample item 'maintain a high level of effort right through to the end of a HIIT session'. The mean of the 6 -items will be calculated.

\section{Brain structure and function}

To further elucidate exercise-induced neural changes, a target subsample of approximately 60 students (ie, 15 students per school from 4 schools; $2 \times i n t e r v e n t i o n$ group, $2 \times$ control group) identified as being in the bottom $50 \%$ of students from their school for CRF (using their baseline PACER test result) will undergo multimodal MRI. We have undertaken a systematic review of neuroimaging studies that have examined associations between physical activity, CRF or muscular fitness, and brain structure/ function. Preliminary findings from this review informed our multimodal MRI protocol to explore changes in the following areas: (i) structural MRI (T1-weighted and T2-weighted imaging) to identify volumetric changes in white and grey matter of the hippocampus, ${ }^{75-77}$ frontal regions/prefrontal cortex ${ }^{75}$ anterior cingulate cortex ${ }^{78}$ and basal ganglia ${ }^{79}$; (ii) diffusion tensor imaging will be used to identify changes in white matter structural connectivity of the superior longitudinal fasciculus and corpus callosum; (iii) resting state functional MRI will be used to assess changes in activation of the default mode network, cognitive control network, saliency network, ${ }^{80}$ hippocampus and prefrontal cortex ${ }^{81}{ }^{82}$; (iv) magnetic resonance spectroscopy will be used to identify changes in brain metabolite concentrations (ie, gamma-aminobutyric acid, N-acetyl aspartate, ATP and glutamate/ glutamine) in the hippocampus and frontal regions/ prefrontal cortex.

\section{On-task behaviour}

Cohort 2 schools will be invited to participate in a substudy to determine the acute effect of the B2L intervention on students' behaviour in the classroom. Classroom observations will be conducted by trained research assistants at baseline and mid-intervention (weeks 5-8) using established methods. ${ }^{83} 84$ During each $30 \mathrm{~min}$ observation period (starting 5 min after students enter the classroom), research assistants will assess the on-task and off-task behaviour of six randomly selected students ( 5 min per student). For each lesson, two observers will randomly select 12 students (ie, 6 boys and 6 girls) and the order in which they are observed (teachers and students will not know who is being observed). Observers will listen to an MP3 audio file via headphones, which will inform them when to observe and record (in $15 \mathrm{~s}$ intervals). After each $10 \mathrm{~s}$ interval, the observers will record the student's behaviour by circling an appropriate code (ie, actively engaged, passively engaged, off-task motor, off-task verbal or off-task passive) using an observation sheet. After $15 \mathrm{~s}$, the observer will then focus on the next student and repeat this process five times until the six students have been observed 20 times. On-task behaviour includes times when the child is actively engaged in an academic activity (eg, reading, writing or performing the designated task) or passively engaged (ie, sitting quietly and listening to the teacher). Off-task behaviour includes times when the student is not engaged in the designated task and can be classified as off-task motor (ie, walking around the class), off-task verbal (ie, talking) or off-task passive (ie, passively not attending to the assigned academic activity). Time spent on-task and off-task during the lesson will be expressed as a percentage of total lessontime. Two trained research assistants will be responsible for conducting all observations and inter-rater reliability scores will be established in the training phase.

\section{Process evaluation}

A detailed process evaluation will be conducted to determine intervention fidelity. Process measures will include: (i) students' mean heart rate from the HIIT sessions (measured using Bluetooth heart rate monitoring technology), (ii) teacher attendance and satisfaction with 
the professional learning workshop and curriculum materials (workshop evaluation questionnaires), (iii) students' satisfaction with all intervention components (student evaluation questionnaire), (iv) number of practical sessions delivered (school champion logs and session observations), (v) teachers' implementation questionnaire (adapted from an existing questionnaire), ${ }^{85}$ (vi) student engagement (objective usage data) with the app and (vii) practical session fidelity (three observations per teacher) using the SAAFE observation checklist. ${ }^{49}$

\section{Statistical analyses}

Analyses of the primary and secondary outcomes will be conducted using linear mixed models in IBM SPSS Statistics for Windows, V.20.0 (IBM, Armonk, New York, USA), with alpha levels set at $\mathrm{p}<0.05$. The models will be used to assess the impact of treatment (B2L or control), time (treated as categorical with levels baseline, 6 and 12 months) and the group-by-time interaction, using random effects to account for the clustered nature of the data. Although randomisation will occur at the school level, statistical analyses will be adjusted for the clustering of effects at the class level, as students from each school are nested in classes. Previous school-based studies have demonstrated that clustering at the school level is negligible after adjusting for clustering at the class level. ${ }^{86} 87$ However, we will test this assumption and additionally adjust our analyses for school-level clustering if required. ${ }^{88}$ Several potential moderators of intervention effects will be explored using interaction tests. ${ }^{89}$ Subgroup analyses will be conducted for the following variables if the significance of the group-by-moderator interaction is $\leq 0.10$ : socioeconomic status (low, medium, high), sex (male, female), baseline weight status (not overweight, overweight/obese), baseline psychological distress (using established cut-offs from the Strengths and Difficulties Questionnaire) and baseline CRF (using FITNESSGRAM fitness standards). Compared with complete-case analyses, mixed models include available data for all participants in the analysis and are thus both more efficient and robust to bias. Mixed model analyses are consistent with the intention-to-treat principle, assuming the data are missing at random. ${ }^{90}$ The validity of this assumption will be explored by assessing relationships between missingness and observed values of covariates and previous outcomes. A range of sensitivity analyses will be conducted (eg, multiple imputation and complete-case analysis). In addition to our primary analysis (ie, intention-to-treat) and sensitivity analyses, we will also conduct two per-protocol analyses (ie, at the class and student levels, respectively). After consideration of typical school disruptions (ie, sporting events, school excursions, exams, etc), we estimate that a minimum of 28 exercise sessions offered over the duration of phases I and II is achievable for schools, and sufficient to observe effects for our primary outcome at the primary end point (ie, 6 months). Therefore, our class-level per-protocol analysis will include only students from classes in which at least 28 school-based sessions were offered. For our student-level per-protocol analysis, we will include only those students who achieved an average heart rate of $80 \%$ of their age-predicted maximum (220-age) across the intervention period (up until our primary end point), using heart-rate data drawn from the B2L smartphone app.

\section{Patient and public involvement}

The need for a time efficient physical activity intervention for older adolescents was identified through consultation with the NSW Department of Education School Sport Unit, who provided initial funding to evaluate the feasibility of the B2L intervention. The pilot study was conducted in two secondary schools in Newcastle $(n=68$ students) and participants (ie, students and teachers) were invited to provide feedback on the intervention and suggestions for further improvement. This feedback was then used to refine the B2L intervention components (eg, B2L smartphone app) and implementation strategies (eg, professional learning for teachers).

The findings of the RCT will be published in peer-reviewed journals and the NSW Department of Education and all participating schools will receive a report outlining the study findings at the conclusion of the trial. Burden of the intervention was not assessed prior to commencing the trial; teacher and student experiences in the intervention will be determined using a detailed process evaluation questionnaire at post-test.

\section{DISCUSSION}

Despite the importance of physical activity and fitness for adolescents' physical and mental health, increasing time demands and academic pressures in the final years of schooling often drive older adolescents to sacrifice time usually spent being active. Although schools are well equipped to promote physical activity to adolescents, secondary schools in NSW do not schedule mandatory physical activity opportunities (eg, physical education, co-curricular school sport) for senior school students (ie, grades 11 and 12). Lack of physical activity may contribute to the high levels of stress, anxiety and depression observed in older adolescents. ${ }^{91-93}$ The B2L intervention will be promoted to schools, teachers, students and parents as a strategy to improve older adolescents' cognitive control and academic performance (rather than focusing on the metabolic health benefits).

Phases I and II of the B2L intervention aim to provide grade 11 students with additional physical activity opportunities embedded within the school day in order to increase fitness and physical activity levels. Phase III will provide an opportunity to explore fitness and behaviour change once the scheduled sessions are no longer facilitated by teachers. We hypothesise that intervention group participants' fitness levels will decrease during this period, but remain higher than control group participants. Providing older adolescents with the knowledge, skills and opportunities to engage in HIIT may compliment 
their participation in other types of leisure-time physical activity. It is therefore important to ensure that students are equipped with the necessary tools in order to engage in self-directed physical activity, outside of the school setting. Self-efficacy (ie, a belief in one's capability or competence within a specific context) is consistently identified as a central determinant of human motivation, and exercise adherence. ${ }^{94}$ B2L has also been guided by self-determination theory and designed to satisfy students' basic psychological needs for autonomy (eg, providing choice/allowing students to feel in control), competence (eg, incorporating technique cards to develop correct exercise form) and relatedness (eg, encouraging social connection and encouragement from others), ultimately impacting on students' autonomous motivation.

Poor implementation may explain why so many schoolbased physical activity interventions fail to reach their potential. Lack of time has been noted as the greatest barrier to implementation by teachers ${ }^{27}$ and providing robust evidence for the positive effects of vigorous physical activity on cognitive and mental health outcomes may provide the impetus for schools to make mandatory physical activity for older adolescents. While several studies have established that HIIT can be successfully delivered in schools, ${ }^{95}$ previous studies have used research staff members or external providers to deliver HIIT sessions, which is neither 'scalable' nor 'sustainable'. Although we do not have funding to conduct an economic evaluation, incorporating short breaks into the school day appears to be a cost-effective way to increase young people's activity levels. $^{96}$

\section{ETHICS AND DISSEMINATION}

School principals, teachers, parents and students all provided informed written consent prior to enrolment. It is not expected that participants will be at any greater risk of adverse events than they would be when participating in other types of school-based physical activity. However, the teacher handbook includes a section for teachers to report any injuries or adverse events that may occur. Any amendments to the study protocols will be publicly available via the Australian and New Zealand Clinical Trials Registry (trial number: ACTRN12615000360516). Data management procedures will be conducted by DRL and SK. All entered data will be de-identified using participant codes and will be stored electronically in a password-protected drive at the University of Newcastle. Quality checks of entered data will be completed by AL (ie, range checks). Access to the final trial dataset will comply with the conditions of the ethics committee approval and will be at the discretion of the lead investigator, DRL.

\footnotetext{
Author affiliations

${ }^{1}$ Priority Research Centre for Physical Activity and Nutrition, University of Newcastle, Callaghan, New South Wales, Australia

${ }^{2}$ Faculty of Education and Arts, University of Newcastle, Callaghan, New South

Wales, Australia

${ }^{3}$ Department of Psychology, Northeastern University, Boston, Massachusetts, USA
}

${ }^{4}$ Department of Physical Therapy, Movement and Rehabilitation Sciences, Northeastern University, Boston, Massachusetts, USA

${ }^{5}$ Centre for Rehab Innovations(CRI), School of Biomedical Sciences and Pharmacy and the Priority Research Centre for Stroke and Brain Injury, Hunter Medical Research Institute (HMRI), University of Newcastle, Callaghan, New South Wales, Australia

${ }^{6}$ Institute for Positive Psychology and Education, Faculty of Health Sciences, Australian Catholic University, Strathfield, New South Wales, Australia

${ }^{7}$ School of Behavioural and Health Sciences, Faculty of Health Sciences, Australian Catholic University, Strathfield, New South Wales, Australia

${ }^{8}$ School of Medicine and Public Health, University of Newcastle, Callaghan, New South Wales, Australia

${ }^{9}$ School of Exercise and Nutrition Sciences, Deakin University, Geelong, Victoria, Australia

${ }^{10}$ School of Biomedical Sciences and Pharmacy, University of Newcastle, Callaghan, New South Wales, Australia

${ }^{11}$ Auckland University of Technology, Human Potential Centre, Auckland, New Zealand

Contributors DRL, CH, PJM, RCP, MNil, CL, NE and JJS secured funding for the project. DRL, NE, JJS, PJM, CL, AAL, MNoe, SAC, and NH designed the intervention. $\mathrm{CH}$, MNil, SRV and DRL designed the multimodal MRI substudy. CH, TTS and AAL designed and contributed to the administration of the cognitive assessments. FRW and PG designed the cortisol measurement protocol. EH conducted the power calculation and guided the statistical analysis. SY and SGK are responsible for project management, school recruitment and data collection. All authors contributed intellectually to the study design and research methodology, or will. AAL and DRL were responsible for drafting the manuscript. All authors provided critical review and endorsed the final version of the manuscript.

Funding The study is funded by the National Health and Medical Research Council (APP1120518) and the NSW Department of Education School Sport Unit.

Competing interests None declared.

Patient consent for publication Not required.

Ethics approval Ethics approval for this study was obtained from the Human Research Ethics Committee of the University of Newcastle, Australia (H-2016-424) and the NSW Department of Education (SERAP: 2017116).

Provenance and peer review Not commissioned; externally peer reviewed.

Open access This is an open access article distributed in accordance with the Creative Commons Attribution Non Commercial (CC BY-NC 4.0) license, which permits others to distribute, remix, adapt, build upon this work non-commercially, and license their derivative works on different terms, provided the original work is properly cited, appropriate credit is given, any changes made indicated, and the use is non-commercial. See: http://creativecommons.org/licenses/by-nc/4.0/.

\section{REFERENCES}

1. Kohl HW, Craig CL, Lambert EV, et al. The pandemic of physical inactivity: global action for public health. Lancet 2012;380:294-305.

2. Hallal PC, Andersen LB, Bull FC, et al. Global physical activity levels: surveillance progress, pitfalls, and prospects. Lancet 2012;380:247-57.

3. Dumith SC, Gigante DP, Domingues MR, et al. Physical activity change during adolescence: a systematic review and a pooled analysis. Int J Epidemiol 2011;40:685-98.

4. Schranz NK, Olds T, Boyd R, et al. Results from Australia's 2016 report card on physical activity for children and youth. J Phys Act Health 2016;13(11 Suppl 2):S87-S94.

5. Hardy LL, Mihrshahi S, Drayton BA, et al. NSW Schools Physical Activity and Nutrition Survey (SPANS) 2015: Full Report. NSW Department of Health, Sydney 2016.

6. Lee DC, Artero EG, Sui X, et al. Mortality trends in the general population: the importance of cardiorespiratory fitness. $J$ Psychopharmacol 2010;24(4 Suppl):27-35.

7. Tomkinson GR, Olds TS, Oliver S. Secular changes in pediatric aerobic fitness test performance: the global picture. Med Sport Sci 2007;50:46-68.

8. Sandercock G, Voss C, McConnell D, et al. Ten year secular declines in the cardiorespiratory fitness of affluent English children are largely independent of changes in body mass index. Arch Dis Child 2010;95:46-7. 
9. Ruiz JR, Rizzo NS, Hurtig-Wennlöf A, et al. Relations of total physica activity and intensity to fitness and fatness in children: the European Youth Heart Study. Am J Clin Nutr 2006;84:299-303.

10. Hay J, Maximova K, Durksen A, et al. Physical activity intensity and cardiometabolic risk in youth. Arch Pediatr Adolesc Med 2012;166:1022-9.

11. Rieck T, Jackson A, Martin SB, et al. Health-related fitness, body mass index, and risk of depression among adolescents. Med Sci Sports Exerc 2013;45:1083-8.

12. Larun L, Nordheim LV, Ekeland E, et al. Exercise in prevention and treatment of anxiety and depression among children and young people. Cochrane Database Syst Rev 2006;3:CD004691.

13. Fedewa AL, Ahn S. The effects of physical activity and physical fitness on children's achievement and cognitive outcomes: a metaanalysis. Res Q Exerc Sport 2011;82:521-35.

14. Singh A, Uijtdewilligen L, Twisk JW, et al. Physical activity and performance at school: a systematic review of the literature including a methodological quality assessment. Arch Pediatr Adolesc Med 2012;166:49-55.

15. Donnelly JE, Hillman $\mathrm{CH}$, Castelli $\mathrm{D}$, et al. Physical activity, fitness, cognitive function, and academic achievement in children: A systematic review. Med Sci Sports Exerc 2016;48:1197-222.

16. Lubans D, Richards J, Hillman C, et al. Physical activity for cognitive and mental health in youth: A systematic review of mechanisms. Pediatrics 2016;138:e20161642.

17. Hills AP, Dengel DR, Lubans DR. Supporting public health priorities: recommendations for physical education and physical activity promotion in schools. Prog Cardiovasc Dis 2015:57:368-74.

18. Metcalf B, Henley W, Wilkin T. Effectiveness of intervention on physical activity of children: systematic review and meta-analysis of controlled trials with objectively measured outcomes (EarlyBird 54). BMJ 2012;345:e5888

19. Dobbins M, Husson H, DeCorby K, et al. School-based physical activity programs for promoting physical activity and fitness in children and adolescents aged 6 to 18. Cochrane Database Syst Rev 2013;19:CD007651.

20. Borde R, Smith JJ, Sutherland R, et al. Methodological considerations and impact of school-based interventions on objectively measured physical activity in adolescents: a systematic review and meta-analysis. Obes Rev 2017;18:476-90.

21. Cohen KE, Morgan PJ, Plotnikoff RC, et al. Physical activity and skills intervention: SCORES cluster randomized controlled trial. Med Sci Sports Exerc 2015;47:765-74.

22. Eather N, Morgan PJ, Lubans DR. Improving the fitness and physical activity levels of primary school children: results of the Fit-4-Fun group randomized controlled trial. Prev Med 2013;56:12-19.

23. Dewar DL, Morgan PJ, Plotnikoff RC, et al. The nutrition and enjoyable activity for teen girls study: a cluster randomized controlled trial. Am J Prev Med 2013;45:313-7.

24. Smith JJ, Morgan PJ, Plotnikoff RC, et al. Smart-phone obesity prevention trial for adolescent boys in low-income communities: the ATLAS RCT. Pediatrics 2014;134:e723-e731.

25. Lubans DR, Morgan PJ, Aguiar EJ, et al. Randomized controlled trial of the Physical Activity Leaders (PALs) program for adolescent boys from disadvantaged secondary schools. Prev Med 2011;52:239-46.

26. Sutherland R, Campbell E, Lubans DR, et al. 'Physical Activity 4 Everyone' school-based intervention to prevent decline in adolescent physical activity levels: 12 month (mid-intervention) report on a cluster randomised trial. Br J Sports Med 2016;50:488-95.

27. Naylor PJ, Nettlefold L, Race D, et al. Implementation of schoo based physical activity interventions: a systematic review. Prev Med 2015;72:95-115

28. Jenkinson KA, Benson A. Barriers to providing physical education and physical education and physical activity in Victorian state secondary schools. Aust J Teach Educ 2010;35:1-17.

29. Weston KS, Wisløff U, Coombes JS. High-intensity interval training in patients with lifestyle-induced cardiometabolic disease: a systematic review and meta-analysis. Br J Sports Med 2014;48.

30. Logan GR, Harris N, Duncan S, et al. A review of adolescent highintensity interval training. Sports Med 2014:44:1071-85.

31. Costigan SA, Eather N, Plotnikoff RC, et al. High-intensity interval training for improving health-related fitness in adolescents: a systematic review and meta-analysis. $\mathrm{Br} J$ Sports $\mathrm{Med}$ 2015;49:1253-61.

32. Biddle SJ, Batterham AM. High-intensity interval exercise training for public health: a big HIT or shall we HIT it on the head?. Int J Behav Nutr Phys Act 2015;12:95

33. Weston KL, Azevedo LB, Bock S, et al. Effect of novel, school-based High-Intensity Interval Training (HIT) on Cardiometabolic health in adolescents: Project FFAB (Fun Fast Activity Blasts) - an exploratory controlled before-and-after trial. PLoS One 2016;11:e0159116.
34. Oliveira BRR, Santos TM, Kilpatrick M, et al. Affective and enjoyment responses in high intensity interval training and continuous training: $A$ systematic review and meta-analysis. PLoS One 2018;13:e0197124.

35. Malik AA, Williams CA, Weston KL, et al. Perceptual responses to high- and moderate-intensity interval exercise in adolescents. Med Sci Sports Exerc 2018;50:1021-30.

36. Leahy AA, Eather N, Smith JJ, et al. Feasibility and preliminary efficacy of a teacher-facilitated high-intensity interval training intervention for older adolescents. Pediatr Exerc Sci 2019;31:107-17.

37. Moher D, Hopewell S, Schulz KF, et al. CONSORT 2010 explanation and elaboration: updated guidelines for reporting parallel group randomised trials. BMJ 2010;340:c869

38. Hoffmann TC, Glasziou PP, Boutron I, et al. Better reporting of interventions: template for intervention description and replication (TIDieR) checklist and guide. BMJ 2014;348:g1687.

39. Cooper Institute for Aerobics Research. Fitnessgram: Test administration manual. Champaign, IL: Human Kinetics, 1999.

40. Heo M, Leon AC. Statistical power and sample size requirements for three level hierarchical cluster randomized trials. Biometrics 2008;64:1256-62.

41. Costigan SA, Eather N, Plotnikoff RC, et al. Preliminary efficacy and feasibility of embedding high intensity interval training into the schoo day: A pilot randomized controlled trial. Prev Med Rep 2015;2:973-9.

42. Australian Bureau of Statistics. Socio-Economic indexes for areas: census of population and housing. Canberra, Australia, 2001.

43. Kennedy SG, Smith JJ, Morgan PJ, et al. Implementing resistance training in secondary schools: a cluster randomized controlled trial. Med Sci Sports Exerc 2018;50:62-72.

44. Lubans DR, Smith JJ, Peralta LR, et al. A school-based intervention incorporating smartphone technology to improve health-related fitness among adolescents: rationale and study protocol for the NEAT and ATLAS 2.0 cluster randomised controlled trial and dissemination study. BMJ Open 2016;6:e010448.

45. Milat AJ, King L, Bauman AE, et al. The concept of scalability: increasing the scale and potential adoption of health promotion interventions into policy and practice. Health Promot Int 2013;28:285-98.

46. Damschroder LJ, Aron DC, Keith RE, et al. Fostering implementation of health services research findings into practice: a consolidated framework for advancing implementation science. Implement Sci 2009;4:50.

47. Beets MW, Okely A, Weaver RG, et al. The theory of expanded, extended, and enhanced opportunities for youth physical activity promotion. Int J Behav Nutr Phys Act 2016;13:120.

48. Deci E, Ryan R. Intrinsic motivation and self-determination in human behaviour. New York: Plenum, 1985.

49. Lubans DR, Lonsdale $\mathrm{C}$, Cohen $\mathrm{K}$, et al. Framework for the design and delivery of organized physical activity sessions for children and adolescents: rationale and description of the 'SAAFE' teaching principles. Int J Behav Nutr Phys Act 2017;14.

50. B Owen K, Smith J, Lubans DR, et al. Self-determined motivation and physical activity in children and adolescents: a systematic review and meta-analysis. Prev Med 2014:67:270-9.

51. Lang JJ, Tomkinson GR, Janssen I, et al. Making a case for cardiorespiratory fitness surveillance among children and youth. Exerc Sport Sci Rev 2018;46:66-75.

52. Mahar MT, Welk GJ, Rowe DA. Estimation of aerobic fitness from PACER performance with and without body mass index. Meas Phys Educ Exerc Sci 2018;22:239-49.

53. Meredith MD, Welk GJ. FitnessGram \& ActivityGram test administration manual (Updated 4th ed. Champaign: IL: Human Kinetics, 2010

54. Chandler JL, Brazendale K, Beets MW, et al. Classification of physical activity intensities using a wrist-worn accelerometer in 8-12-year-old children. Pediatr Obes 2016;11:120-7.

55. Castro-Piñero J, Ortega FB, Artero EG, et al. Assessing muscular strength in youth: usefulness of standing long jump as a general index of muscular fitness. J Strength Cond Res 2010;24:1810-7.

56. Lubans DR, Morgan P, Callister R, et al. Test-retest reliability of a battery of field-based health-related fitness measures for adolescents. J Sports Sci 2011;29:685-93.

57. Ruiz JR, Castro-Piñero J, España-Romero V, et al. Field-based fitness assessment in young people: the ALPHA health-related fitness test battery for children and adolescents. Br J Sports Med 2011;45:518-24.

58. Cole TJ, Lobstein T. Extended international (IOTF) body mass index cut-offs for thinness, overweight and obesity. Pediatr Obes 2012;7:284-94.

59. Peirce JW. PsychoPy-Psychophysics software in Python. J Neurosci Methods 2007;162(1-2):8-13. 
60. Weintraub S, Dikmen SS, Heaton RK, et al. Cognition assessment using the NIH Toolbox. Neurology 2013;80(11 Suppl 3):S54-S64.

61. Jaeggi SM, Buschkuehl M, Perrig WJ, et al. The concurrent validity of the $\mathrm{N}$-back task as a working memory measure. Memory 2010;18:394-412.

62. Diamond A. Executive functions. Annu Rev Psychol 2013;64:135-68

63. Haatveit BC, Sundet K, Hugdahl K, et al. The validity of $d$ prime as a working memory index: results from the "Bergen $n$-back task". J Clin Exp Neuropsychol 2010;32:871-80.

64. Gerber M, Jonsdottir IH, Kalak N, et al. Objectively assessed physical activity is associated with increased hair cortisol content in young adults. Stress 2013;16:593-9.

65. Stalder T, Steudte S, Miller R, et al. Intraindividual stability of hair cortisol concentrations. Psychoneuroendocrinology 2012;37:602-10.

66. Dettenborn L, Tietze A, Bruckner F, et al. Higher cortisol content in hair among long-term unemployed individuals compared to controls. Psychoneuroendocrinology 2010;35:1404-9.

67. Van Uum SH, Sauvé B, Fraser LA, et al. Elevated content of cortisol in hair of patients with severe chronic pain: a novel biomarker for stress. Stress 2008;11:483-8.

68. Rietschel L, Streit F, Zhu G, et al. Hair cortisol and its association with psychological risk factors for psychiatric disorders: a pilot study in adolescent twins. Twin Res Hum Genet 2016;19:438-46.

69. Cohen S, Kamarck T, Mermelstein R. A global measure of perceived stress. J Health Soc Behav 1983;24:385-96.

70. Mellor D. Normative data for the strengths and difficulties questionnaire in Australia. Aust Psychol 2005;40:215-22.

71. Markland D, Tobin V. A modification to the behavioural regulation in exercise questionnaire to include an assessment of amotivation. Journal of Sport and Exercise Psychology 2004;26:191-6.

72. Emm-Collison LG, Standage M, Gillison FB. Development and Validation of the Adolescent Psychological Need Support in Exercise Questionnaire. J Sport Exerc Psychol 2016;38:505-20.

73. Tennant R, Hiller L, Fishwick R, et al. The Warwick-Edinburgh Mental Well-being Scale (WEMWBS): development and UK validation. Health Qual Life Outcomes 2007;5:63.

74. Sánchez-López M, Martínez-Vizcaíno V, García-Hermoso A, et al. Construct validity and test-retest reliability of the International Fitness Scale (IFIS) in Spanish children aged 9-12 years. Scand J Med Sci Sports 2015;25:543-51.

75. Esteban-Cornejo I, Cadenas-Sanchez C, Contreras-Rodriguez O, et al. A whole brain volumetric approach in overweight/obese children: Examining the association with different physical fitness components and academic performance. The ActiveBrains project. Neuroimage 2017;159:346-54.

76. Ortega FB, Campos D, Cadenas-Sanchez C, et al. Physical fitness and shapes of subcortical brain structures in children. Br J Nutr 2017:33:1-10.

77. Chaddock L, Erickson KI, Prakash RS, et al. A neuroimaging investigation of the association between aerobic fitness, hippocampal volume, and memory performance in preadolescent children. Brain Res 2010;1358:172-83.

78. Krafft CE, Schwarz NF, Chi L, et al. An 8-month randomized controlled exercise trial alters brain activation during cognitive tasks in overweight children. Obesity 2014;22:232-42.

79. Chaddock L, Hillman CH, Pontifex MB, et al. Childhood aerobic fitness predicts cognitive performance one year later. J Sports Sci 2012;30:421-30.

80. Krafft CE, Pierce JE, Schwarz NF, et al. An eight month randomized controlled exercise intervention alters resting state synchrony in overweight children. Neuroscience 2014;256:445-55.
81. Chaddock-Heyman L, Erickson KI, Voss MW, et al. The effects of physical activity on functional MRI activation associated with cognitive control in children: a randomized controlled intervention. Front Hum Neurosci 2013;7:72.

82. Davis CL, Tomporowski PD, McDowell JE, et al. Exercise improves executive function and achievement and alters brain activation in overweight children: a randomized, controlled trial. Health Psychol 2011;30:91-8.

83. Alberto PA, Troutman AC. Applied Behavior Analysis for Teachers. Australia: Pearson, 2003.

84. Riley N, Lubans DR, Holmes K, et al. Findings from the EASY Minds cluster randomized controlled trial: evaluation of a physical activity integration program for mathematics in primary schools. J Phys Act Health 2016;13:198-206.

85. Carlson JA, Engelberg JK, Cain KL, et al. Contextual factors related to implementation of classroom physical activity breaks. Trans/ Behav Med 2017;7:581-92.

86. Aelterman $\mathrm{N}$, Vansteenkiste M, Van Keer H, et al. Students' objectively measured physical activity levels and engagement as a function of between-class and between-student differences in motivation toward physical education. $J$ Sport Exerc Psychol 2012;34:457-80.

87. Lonsdale C, Rosenkranz RR, Sanders T, et al. A cluster randomized controlled trial of strategies to increase adolescents' physical activity and motivation in physical education: results of the Motivating Active Learning in Physical Education (MALP) trial. Prev Med 2013;57:696-702.

88. Lonsdale C, Lester A, Owen KB, et al. An internet-supported school physical activity intervention in low socioeconomic status communities: results from the Activity and Motivation in Physical Education (AMPED) cluster randomised controlled trial. Br J Sports Med 2019;53:341-7.

89. Yildirim M, van Stralen MM, Chinapaw MJ, et al. For whom and under what circumstances do school-based energy balance behavior interventions work? Systematic review on moderators. Int J Pediatr Obes 2011;6(2-2):e46-e57.

90. White IR, Carpenter J, Horton NJ. Including all individuals is not enough: Lessons for intention-to-treat analysis. Clin Trials 2012;9:396-407.

91. Australian Institute of Health and Welfare. Young Australians: their health and wellbeing 2007. Canberra: AlHW, 2007. Cat. No. PHE 87.

92. North BW. Under Pressure: Senior Students in High Stakes Assessment: University of New South Wales, 2015.

93. Australian Bureau of Statistics. (2013). Children's participation in cultural and leisure activities. Retrieved from http://www.abs.gov. au/ausstats/abs@.nsf/products/4901.0 apr+2012 main+features internet+and+mobile+phones (4 Feb 2013).

94. Bandura A. Human agency in social cognitive theory. Am Psychol 1989;44:1175-84.

95. Bond B, Weston KL, Williams CA, et al. Perspectives on highintensity interval exercise for health promotion in children and adolescents. Open Access J Sports Med 2017;8:243-65.

96. Babey SH, Wu S, Cohen D. How can schools help youth increase physical activity? An economic analysis comparing school-based programs. Prev Med 2014;69(Suppl 1):S55-S60.

97. Carlson JA, Engelberg JK, Cain KL, et al. Implementing classroom physical activity breaks: Associations with student physical activity and classroom behavior. Prev Med 2015;81:67-72. 